\title{
The utility of digital breast tomosynthesis in axillary lymph node post clip
} mammography

Regional breast cancer staging with axillary lymph node biopsy has been in the spotlight in recent years following results of the American College of Surgeons Oncology Group (ACOSOG) Z1011 trial. Subsequent studies have demonstrated that clip marker placement in biopsy proven metastatic axillary lymph nodes aids in targeted axillary lymph node dissection, ensuring excision of known metastatic disease and reducing the false negative rate of sentinel lymph node biopsy. We believe the use of DBT for documentation of axillary lymph node clip marker placement presents an opportunity to expand on the established benefits of this new technology. DBT can provide three-dimensional assessment of axillary clip marker placement, assists future follow-up and localization, improves workflow efficiency, helps potentially reduce patient dose and enhances the patient experience.

KEYWORDS: DBT - mammography - chemotherapy

\section{Introduction}

Digital breast tomosynthesis (DBT) has become a widely accepted adjunct to conventional full field digital mammography in the evaluation and management of breast disease. With its use in screening mammography, studies have demonstrated an overall increase in cancer detection rate, with the detection of smaller cancers and reduction in the rate of falsepositive findings and associated call-backs [1]. DBT has also been shown to be superior to twodimensional digital mammography in detecting architectural distortion and other abnormalities, irrespective of breast density [2]. In recent years, the use of DBT technology has expanded beyond screening and diagnostic mammography to also include use in needle localization and tomosynthesis-guided biopsy procedures [3].

With its increasing utilization in breast imaging, a new role has emerged for DBT in the post-biopsy clip setting. Historically, patients undergoing image guided breast biopsy have a clip marker deployed during the procedures. The purpose of marker clip placement is threefold; it allows documentation of image guided biopsy intervention, provides a mammographic correlate for lesions biopsied under ultrasound and MRI guidance, and allows for detection and documentation of biopsy proven malignancies that subsequently undergo surgical excision with or without neoadjuvant chemotherapy $[4,5]$. Several studies have examined the use of DBT in documenting clip marker placement following ultrasound guided breast biopsy, on demonstrating that DBT was superior to FFDM alone when assessing clip marker positioning and possible displacement [4]. In this manuscript, we would like to propose a potential new role for DBT in evaluating post biopsy clip placement following biopsy of metastatic axillary level I lymph nodes.

Regional breast cancer staging with axillary lymph node biopsy has been in the spotlight in recent years [5-7] following results of the American College of Surgeons Oncology Group (ACOSOG) Z0011 trial. This randomized, multicenter trial demonstrated that in a specific cohort of patients, axillary lymph node dissection (ALD) provides no additional survival benefit over sentinel lymph node dissection (SLD) [7]. Subsequent studies have demonstrated that clip marker placement in biopsy proven metastatic axillary lymph nodes aids in targeted axillary lymph node dissection, ensuring excision of known metastatic disease and reducing the false negative rate of SLD [7]. A successful targeted SLD provides survival benefit while sparing patients the associated increased morbidity associated with full ALD [7]. At our institution, the initial diagnostic evaluation of a patient with suspected breast cancer includes ultrasound evaluation of the regional nodal basins [8]. When metastatic disease is confined to the ipsilateral axillary level I or level II lymph nodes (N2 disease), the index (most suspicious) axillary level I lymph node is biopsied and a clip marker placed. These clipped lymph nodes can be more confidently localized during targeted axillary nodal dissection with intraoperative specimen radiography performed to document successful excision of the clipped node. This can also be used in tandem with sentinel lymph node biopsy to assess the accuracy of targeted sentinel node biopsy. The Z1011 trial [7] substantiated the efficacy of clip marker lacement in these
Toma S Omofoye, Jay R Parikh \& Megan Kalambo* Department of Radiology, The University of Texas MD Anderson Cancer Center, Houston, TX *Author for correspondence: 
axillary nodes demonstrating that up to $20 \%$ of biopsy proven metastatic lymph nodes did not correspond to the sentinel lymph node by Technetium-labeled lymphoscintigraphy $[5,6]$.

Clip marker placement within the biopsy proven metastatic lymph node is also important to follow in cases where response to neoadjuvant chemotherapy is being assessed. In the absence of a clip marker, these lymph nodes may become less readily detectable by imaging following treatment. Currently, routine documentation of clip position is accomplished by ultrasound (by obtaining a single or orthogonal views of the biopsy clip within the targeted lesion), followed by orthogonal mammographic views in the cranio-caudal (CC) and true lateral (LM) position. As the axilla is often excluded on a standard CC view, and only a portion of the axilla visualized on the $\mathrm{LM}$ view, technologists will obtain a single mediolateral oblique (MLO) view to document clip marker placement in an axillary lymph node. The end result is that axillary lymph node post-biopsy clips are often documented in a single mammographic view despite obtaining images in multiple planes.
This makes it difficult to determine whether the clip marker truly sits in the biopsied lymph node rather than along the plane in a single view. Imaging in a single plane only assesses placement in a single plane and cannot document lateral or medial migration.

\section{Case}

The need for biplanar and orthogonal documentation of clip markers placed in the axilla represents a unique opportunity to expand on the utility of DBT. DBT technology, by obtaining a series of low dose images across a 30 degree arc with 9-25 separate projections, permits reconstruction of multiple parallel planes, each plane in focus with out-of-plane tissues blurred [1]. In our sample case, a 77 year old woman presented to the breast clinic with a palpable finding in her right breast. This correlated with an irregular $5 \mathrm{~cm}$ mass on mammography obtained in $2 \mathrm{D} / 3 \mathrm{D}$ combination view (FIGURE 1). Sonography demonstrated the breast mass as well as a single abnormal ipsilateral axillary level I lymph node that underwent FNA biopsy and placement of a "coil" shaped clip marker (FIGURE 2).

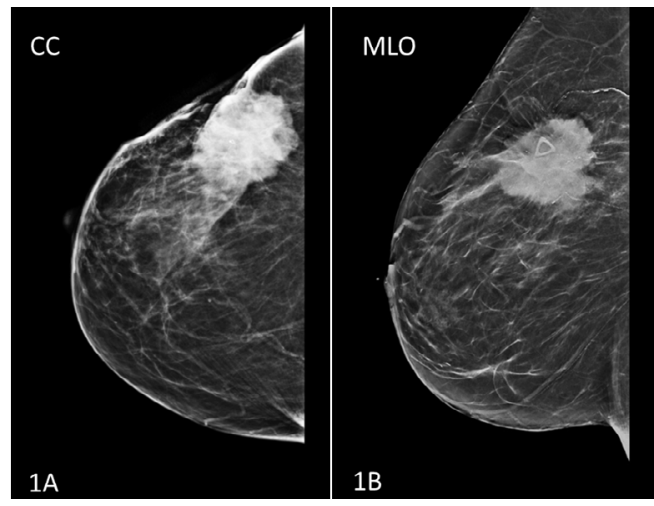

Figure 1. 77 years old female presents with a palpable finding in the right breast. CC and MLO synthetic mammogram views demonstrate a $5 \mathrm{~cm}$ irregular mass with spiculated margin in the right breast upper outer quadrant. There is associated architectural distortion, overlying skin thickening and retraction.
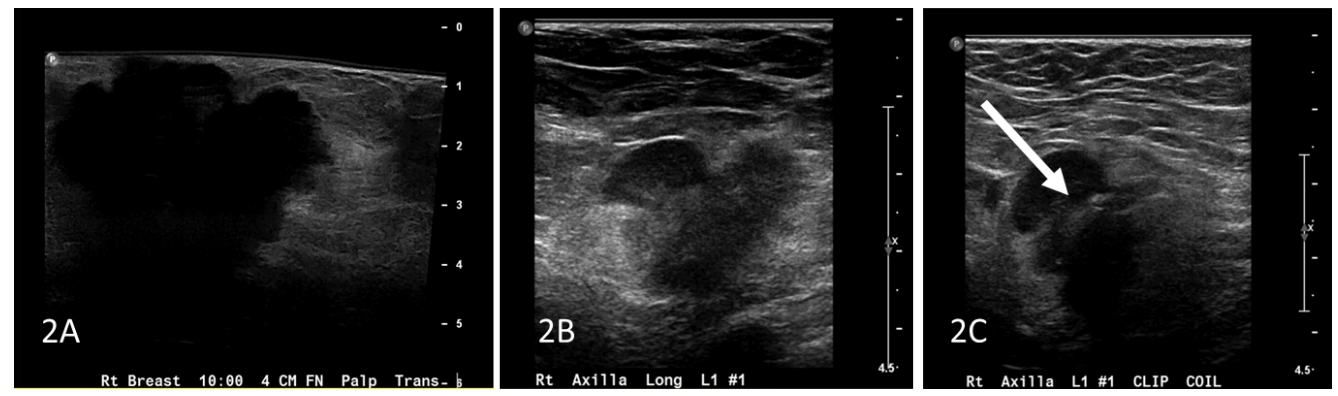

Figure 2. Ultrasound demonstrates an irregular hypo echoic mass in the right breast (2A) corresponding with the area of palpable concern. A single abnormal appearing axillary level I lymph node is also noted (2B). Ultrasound guided FNA biopsy of the targeted axillary lymph node (2C) was performed followed by placement of a coil shaped clip (long arrow). 

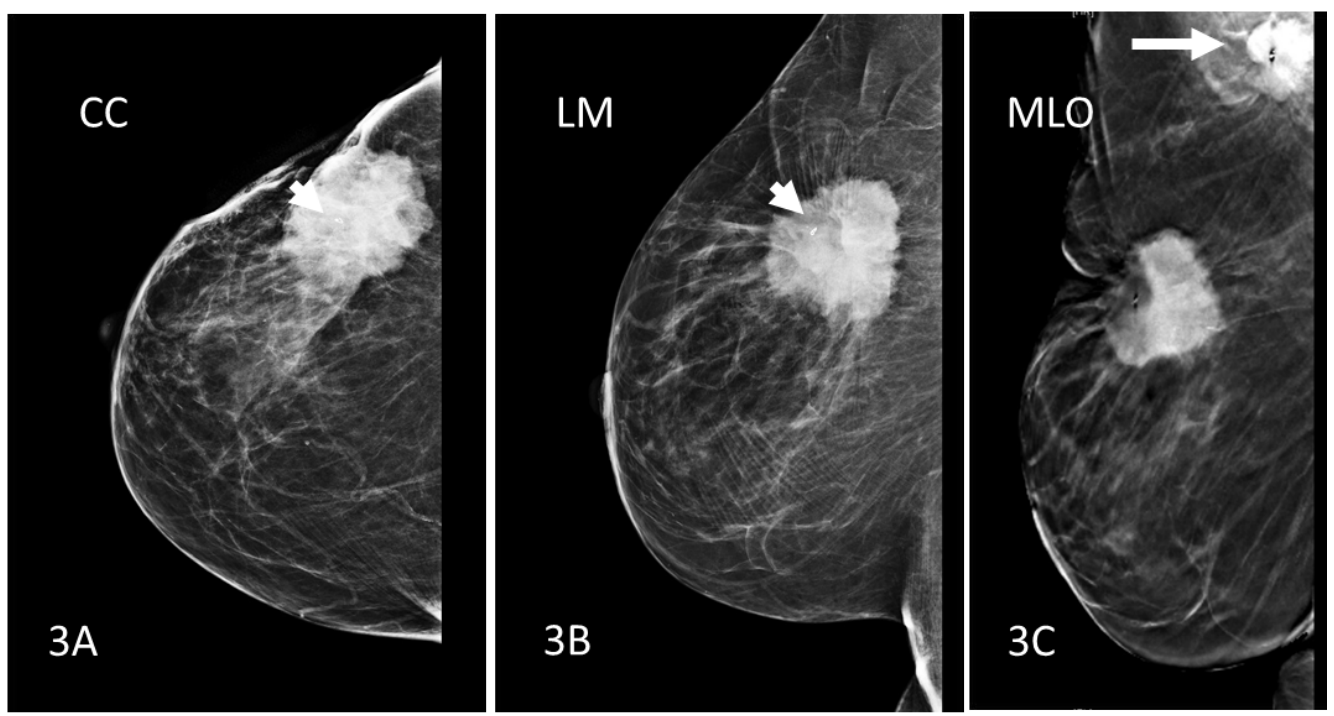

Figure 3. Post-biopsy clip CC and LM synthetic mammogram and tomosynthesis images demonstrate satisfactory positioning of the "wing-shaped" clip in the right breast (arrowhead, 3A and 3B). The low axillary nodal basin is not visualized on these two standard imaging views. Dedicated MLO tomosynthesis imaging demonstrates satisfactory positioning of the post-biopsy clip in the right axillary lymph node. Notice the radio-lucent halo around the axillary lymph node and post-biopsy indicating in-plane imaging (3C, arrow).

Post procedure imaging was performed in standard CC and LM views with inadequate visualization of the axilla. Therefore, a dedicated MLO tomosynthesis view was obtained with demonstration of accurate positioning of the post-biopsy clip in the axillary lymph node (FIGURE 3).

\section{Results}

The use of DBT for accurately localizing post biopsy clips in the axilla has several important implications for the practice of breast imaging. There is likely to be improved work flow-when an axillary lymph node is biopsied, documentation can take the form of MLO imaging only obtained with 3D technology. When both a breast lesion and axillary lesion need to be visualized, imaging can be obtained in CC and MLO views with DBT. This would effectively eliminate the need for a true lateral view in most instances. It also streamlines the process for mammography technologists who, in attempting to capture axillary clips on lateral views may take multiple images in that view, before attempting MLO views. The time saved for the technologist and quicker mammography room turnover for the department represent efficiencies in workflow.

DBT imaging of the axilla for post biopsy clips simultaneously enhances patient safety by documenting satisfactory clip marker placement in two planes and the patient experience by reducing the number of post-biopsy views required for documentation. Given heightened physician and patient awareness of radiation dose, principles of ALARA and the Image wisely endeavor [9], the ability to eliminate any redundant imaging is of paramount importance. The elimination of the true lateral view results in an overall decrease in patient radiation exposure. In the future, clinicians may be interested in examining the value of standard views (CC and LM) versus DBT $\mathrm{CC}$ and MLO views versus DBT MLO imaging only. Additionally, as practice transition from 2D/3D combination imaging towards synthetic imaging with composite views, patient dose is further reduced. Some researchers have evaluated measuring clip displacement on tomosynthesis [4] and this can be applied to the axilla. For patients, the biopsy day is often fraught with anxiety and discomfort and a more streamlined, efficient, post-biopsy experience with appropriate postbiopsy DBT image documentation involving less physical manipulation of a recently biopsied site would be a welcome improvement. The overall improved experience will likely be reflected in improved patient satisfaction.

The use of DBT for documentation of axillary lymph node clip marker placement presents an opportunity to expand on the established benefits of this new technology. DBT provides three-dimensional assessment of axillary clip 
marker placement, assists future follow-up and localization, improves workflow efficiency, helps potentially reduce patient dose and enhances the patient experience.

\section{REFERENCES}

1. Friedewald SM, Rafferty EA, Rose SL et al. Breast cancer screening using tomosynthesis in combination with digital mammography. JAMA. 311, 2499-2507 (2014).

2. Dibble EH, Lourenco AP, Baird GL et al. Comparison of digital mammography and digital breast tomosynthesis in the detection of architectural distortion. Eur. Radiol. 28, 3-10 (2018).

3. Omofoye TS, Martaindale S, Teichgraeber DC. Implementation of upright digital breast tomosynthesis-guided stereotactic biopsy. Acad.
Radiol. 24, 1451-1455 (2017).

4. Schul WR, Dankerl P, Dilbat G et al. Comparison of sonography versus digital breast tomosynthesis to locate intra-mammary marker clips. Geburtshilfe. Frauenheilkd. 75, 72-76 (2015).

5. Shin K, Caudle AS, Kuerer HM et al. Radiologic mapping for targeted axillary dissection: Needle biopsy to excision. AJR. Am. J. Roentgenol. 207, 1372-1379 (2016).

6. Caudle AS, Yang WT, Mittendorf EA et al. Selective surgical localization of axillary lymph nodes containing metastases in patients with breast cancer: A prospective feasibility trial.
JAMA. Surg. 150, 137-143 (2015).

7. Giuliano AE, Hunt KK, Ballman KV et al. Axillary dissection vs. no axillary dissection in women with invasive breast cancer and sentinel node metastasis: A randomized clinical trial. JAMA. 305, 569-575 (2011).

8. Fornage BD. Local and regional staging of invasive breast cancer with sonography: 25 years of practice at MD Anderson Cancer Center. Oncologist. 19, 5-15 (2014).

9. Mayo SWW, Morin RL. Image wisely: The beginning, current status and future opportunities. J. Am. Coll. Radiol. 14, 442-443 (2017). 